
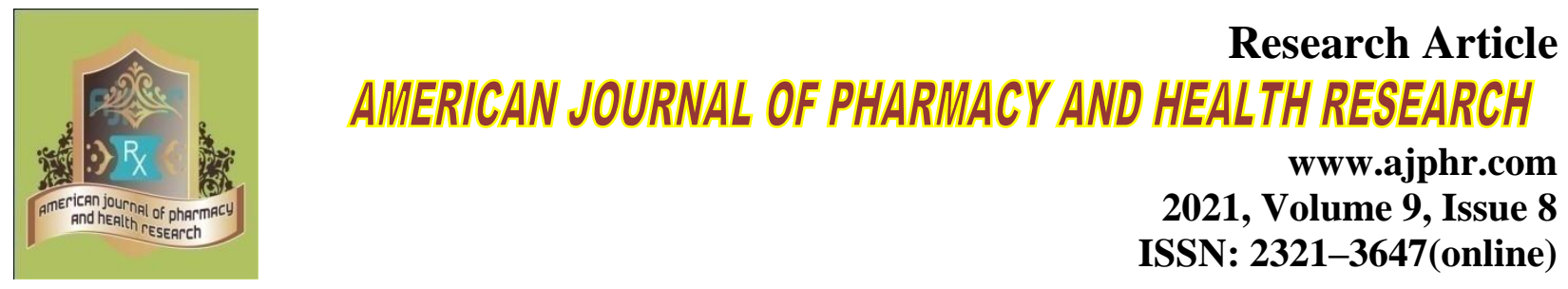

\title{
Study on Evaluation of Medication Adherence and Drug Related Problems In High Risk Elderly Population
}

\author{
Binu KM*, Binu Prasad, H. Doddayya \\ Department of Pharmacy Practice, NET Pharmacy College, Raichur-584103 Karnataka, India
}

\begin{abstract}
Medication adherence is a crucial component in the treatment of chronic diseases. In the elderly, clinicians are faced with a unique set of problems associated with adherence that they may not have been adequately trained for. The present study was designed to assess the sociodemographic profile, adherence and drug-related problems among elderly. Methods: An observational study involving 30 patients aged over 60 years using at least five prescription drugs and discharged from hospital. The occurrence of adherence and DRP were assessed by means of an identification tool specifically developed for use by community pharmacists, including a semi structured patient interview and a checklist of common DRP. Results: Out of 30 study subjects, it was observed that majority of the study subjects had drug related problems. High, moderate and low adherence to medication was found. Conclusion: It is evident that there is a great need on the part of health care providers to not only prescribe medications to the patients but also make a conscious effort to address the issue of non-adherence to the prescribed medications and other non-pharmacological measures.
\end{abstract}

Keywords: Morisky, Drug compliance, elderly, habits, medications 


\section{INTRODUCTION}

Geriatric population is more prone for various chronic and recurrent illnesses which may require chronic medication with multiple drugs. The process of aging involves progressive and irreversible decline in organ function that occurs over time even in the absence of injury, illness, or poor lifestyle like unhealthy diet, lack of exercise, substance abuse, etc.,. Currently the aging population is rapidly increasing, particularly in developing regions of the world. Because of the chronic nature of the disease, the need for multiple drug therapy with complex medication regime, increasing cost of therapy, adverse effects, drug interactions, forgetfulness, lack of familial and social support and care, elderly patients may not be fully compliant to long term medications. ${ }^{1}$

The global population is aging. In 1950, there were 205 million people aged 60 years and older and this number is expected to increase to nearly 2 billion in 2050. Particularly for the treatment of chronic diseases, elderly patients were found to use about three times more drugs than younger patients. They are therefore at a higher risk of experiencing drug-related problems. In general only $50 \%$ of general population has been estimated to adhere to their medications, and this may range from 47 to $100 \%$ in elderly. Poor compliance among older persons is a public health concern. This poses a greater responsibility on the health services especially in developing countries like India; where there is a greater strain on available health infrastructure and delivery systems. Numerous studies have been done in Indian population to assess the problem and the extent of medication compliance in geriatric patients and to analyse the various factors contributing the human and economic costs associated with nonadherence. ${ }^{2}$

Pharmacologic therapy is a key component in the treatment of chronic diseases. However, about $50 \%$ of patients with chronic diseases do not take medication as prescribed. In chronic diseases that require long-term treatment, low medication adherence usually leads to poor clinical outcome, drug-related side effects, and increased social health care costs. Moreover, it has been established that the proper use of medication is one of the important key factors for the selfmanagement of most chronic diseases. Therefore, it is important to improve medication adherence in patients with chronic diseases to minimize early deaths and social burden. ${ }^{3}$

The term adherence is defined as the extent to which a person's behavior of taking medication, following diet, and/or executing lifestyle changes corresponds with agreed recommendations from the health care provider. Adherence rates are typically higher among patients with acute conditions, as compared with those with chronic conditions; persistence among patients with chronic conditions is disappointingly low, dropping most dramatically after the first 6 months of 
therapy. Medication adherence is influenced by many different factors, such as medication factors, patient factors, physician factors, system-based factors, and so on. ${ }^{4}$

Nonadherence is a growing concern to clinicians and health-care systems. Nonadherence behaviors broadly fall into two categories: 1) unintended nonadherence, which occurs due to forgetfulness, bad eyesight, and difficulty in movement and 2) intentional nonadherence, which occurs when medication is deliberately avoided, despite the ability to take the medication. Intentional nonadherence arises when a patient decides not to take the treatment as instructed. To increase medication adherence, it is critical to understand the underlying reason for nonadherence. ${ }^{5,6}$

Low adherence to prescribed medication in patients with non-communicable diseases (NCDs) increases risk of hospitalization, development of complication and premature mortality. Duration of the disease also bears a direct effect on the adherence of the patient to the treatment. The decline in adherence is most rapid after the first 6 months of therapy. Especially, the consequences of drug noncompliance may be serious in older patients. Some older patients who are acutely ill may take more than the prescribed dose of a medication in the mistaken belief that more of the drug will speed their recovery. Such overuse has clearly been associated with adverse drug effects. The use of at least three drugs, and often more, is common in the elderly, with estimates of as many as $25 \%$ of older people taking at least three drugs. ${ }^{7}$

Studies have assessed the frequency and specific nature of DRP among older patients with multiple drug use discharged from hospital. DRP include contraindications, drug-drug interactions, adverse drug reactions, prescription errors, and noncompliance with drug use. With respect to older patients with comorbidities and using multiple drugs, DRP are associated with an increased risk of hospital readmissions, morbidity, and mortality. Discharge of patients from the hospital setting to home care is another important risk factor for DRP. Lack of continuity of care and discontinuity of medication following inadequate transfer of information between hospital and primary care, especially in the event of changes in the drug regimen, were found to be major underlying causes. ${ }^{8}$

In the case of elderly living alone, family support is absent, resulting in a greater chance of sustained condition of chronic diseases, both physically and mentally. Indeed, the level of physical and mental health of elderly living alone is lower than those living with family members. In order to develop effective interventions to identify and address these DRP, it is very important to get more insight into the specific nature of DRP in this specific patient group and determinants of their occurrence. The effect of determinants on the occurrence of these DRP was 
also studied seems to be higher with increased age. ${ }^{9}$

In this context the department of Pharmacy practice has proposed the study entitled "Study on Evaluation of Medication Adherence and Drug Related problems in High Risk Elderly Population.

\section{MATERIALS AND METHOD}

The study was carried out for a period of three months. A prospective observational study was carried out by 30 patients using a questionnaire and direct interactions with patients were also done to comply the data needed for assessment. The study was started after taking consent from respective authorities. Elderly patients of either gender aged 65 years and older and patients using five or more chronic drugs were selected The instrument used in the study is a three part questionnaire. Section-1 deals with socio-demographic characteristics of respondent like age, family and marital status. Section-2 includes Morisky Medication Adherence Scale (MMAS-8Item) which was used to measure the medication adherence among the subjects. Section-3 deals with the factors associated with drug related problems which consists of Socio and demographic factors, Behavioral factors, Co morbidity related factors, Therapy and system related factors, Support related factors, Patient related factors.

\section{RESULTS AND DISCUSSION}

As a result, a total of 30 patients were included for the analysis. Of them, $83 \%$ were women. Almost half of the participants (55.2\%) were between the ages of 75 and 84 years. Moreover, $16 \%$ were widows, 8 were not educated, and 3\% WERE SEPARATED. Of the total, study subjects who had used alcohol $28 \%$ and those who had used tobacco were $8 \%$, smoking $28 \%$ constituted the majority.

Morisky et al found that knowledge, patient satisfaction, coping skills, stress level, and medication complexity are associated with adherence. Cognitive factors like memory deficit among subjects were another factor which was significantly associated with high medication adherence. Patients who reported memory deficit were found to be poor adherent compared to others. $40 \%$ of the patients possessed the Memory loss and other complex cognitive disorders.

The complex process of medication intake is severely influenced by the impairment in multiple cognitive functions. Majority (68\%) of the patients have forgotten to take their medications on the previous day. Other than these, elderly patients often found difficulty remembering to take all their medications such as $4 \%$ all the time, $56 \%$ sometimes or usually, $28 \%$ once in a while. 
Seven days reminder box or instrumented pills are some of the strategies to tackle such issues. Hence, making them understand the early signs of degeneration and taking adequate measures to make the elderly cope with their disabilities are very crucial in maintaining their compliance.

This study shows that DRP occur frequently among elderly patients discharged from hospital and using several drugs to treat chronic diseases. The number of DRP was also significantly associated with the number of drugs prescribed shown by Ahamad et al.

Polypharmacy is a major risk factor in experiencing DRP. Discharge of elderly patients from hospital using five or more drugs for treatment of chronic disease is associated with DRP. As expected, the number of DRP per patient increased with an increase in the number of drugs used. $76 \%$ have 7 or more medications in their current regular use.

In this study, $68 \%$ patients shown that the proportion of adherence was hindered by medications. More than half of the patients were reported to have experienced at least one side effect. This percentage is considerably higher than that found in other studies of elderly patients discharged from hospital. The difference can be explained by the number of drugs used by each patient. The high prevalence of side effects also corroborates the results of Creswell et al, who showed that the number of drugs used by older patients was a risk factor for occurrence of side effects.

Not knowing the purpose and use of at least one of their medicines and fear of side effects were also DRP frequently reported by patients. this was reported by not less than $40 \%$ of patients. These issues therefore should be specifically addressed when patients are counselled on their medication. 
Do you ever feel hassled about sticking to your treatment plan?

do you sometimes stop taking your medicine?

Do you take your medicines yesterday

do you sometimes forget to bring along your medications?

Have you ever cut back or stopped taking your medication

were there any days when you did not take your medicine

Do you sometimes forget to take your pills?

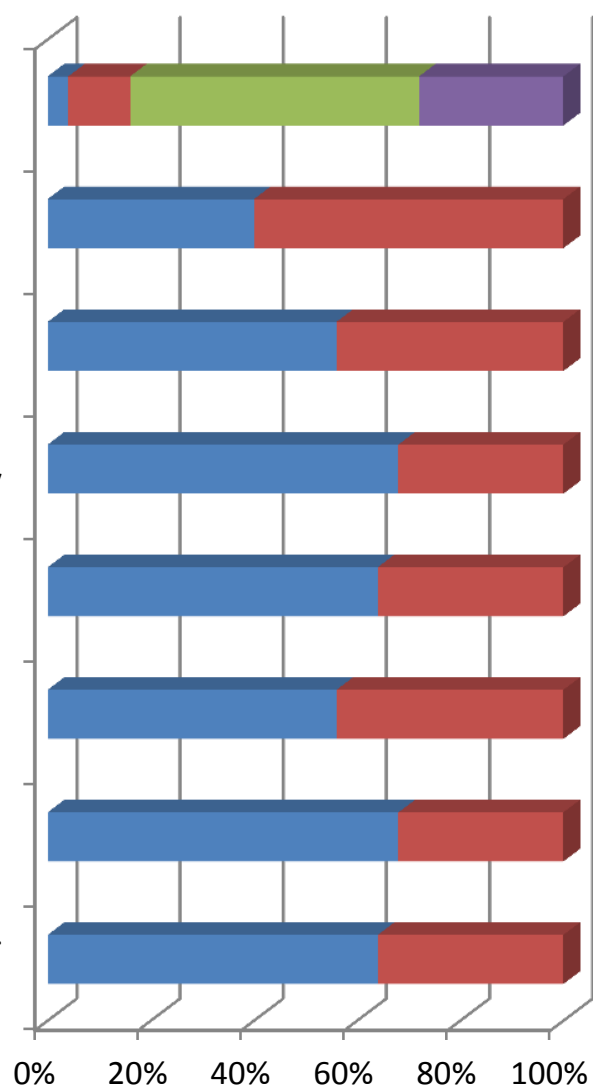




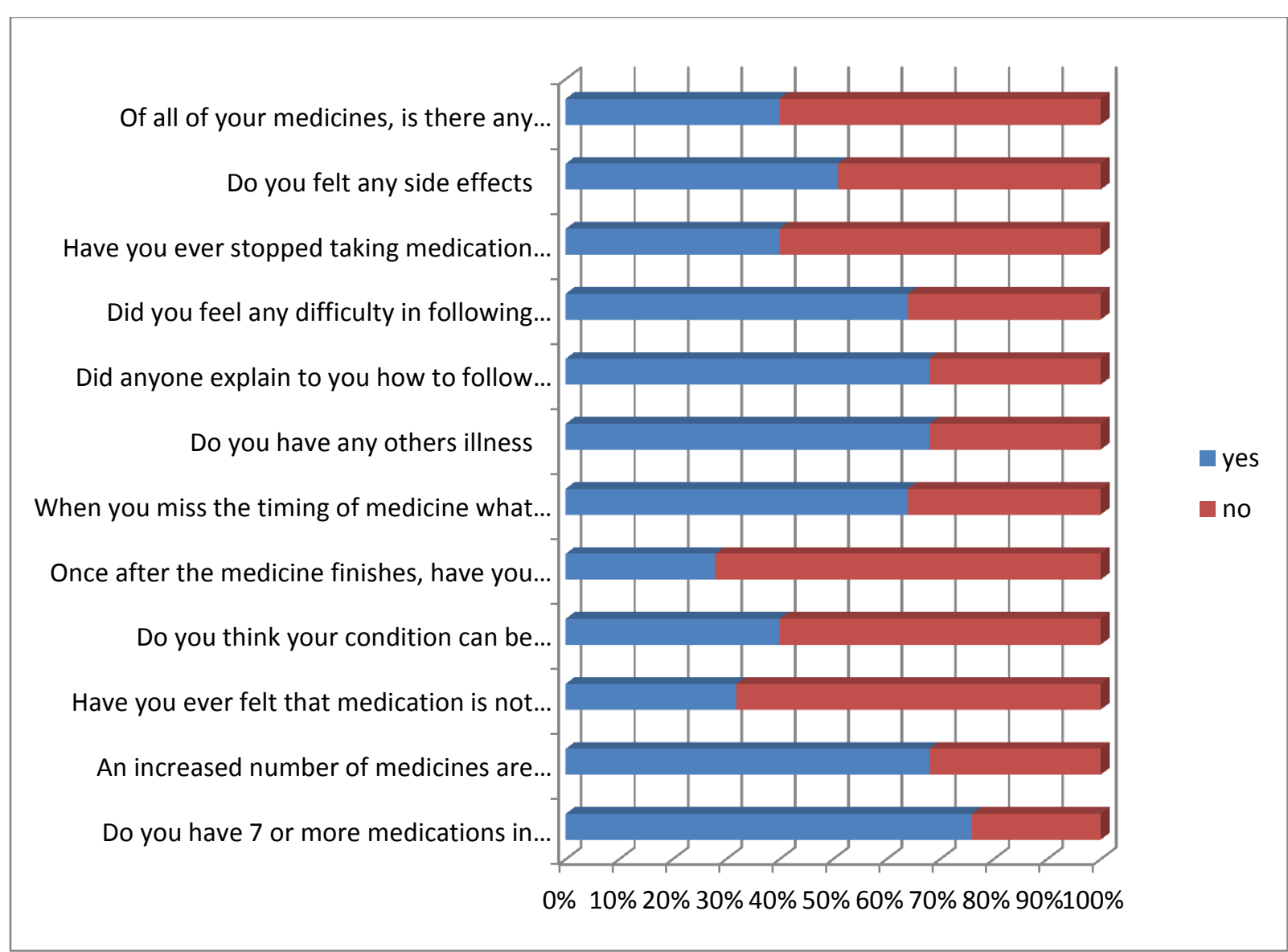

Figure 2: Drug Related Problems

Table 1: socio-demographics

\section{CONCLUSION}

\begin{tabular}{llll}
\hline S.No & Factors & & Percentage \\
\hline 1. & Habits & Tea & $76 \%$ \\
& & Coffee & $68 \%$ \\
& & Alcohol & $28 \%$ \\
& & Tobacco & $8 \%$ \\
& & Smoking & $28 \%$ \\
& & Dust & $4 \%$ \\
2. & Allergy & Yes & $12 \%$ \\
& & No & $88 \%$ \\
\hline
\end{tabular}

Adherence to medications is important in ensuring the therapeutic benefits delivered to patients. However, adherence to medications has always been an issue, especially amongst the elderly. Adherence rates can be improved by disease and treatment specific instructions to the patients along with adequate motivation and constant reinforcement by the health care providers. This study confirms the existence of poor compliance to the prescribed medication among geriatric subjects with chronic diseases. Various considerations have to be taken while prescribing regimens for better drug compliance from the patient side. The importance of taking drugs 
regularly has to be re-emphasized for the benefit of the patient. Particularly the patient group included in the present study, occurrence of DRP may result in increased risk of hospital readmission, morbidity, mortality, and health care costs. An important task for community pharmacists is therefore to identify, resolve, and prevent the occurrence of DRP in this group of patients which, in the coming years, is expected to grow considerably in size. Using comprehensive tools to identify DRP, it is very important to develop intervention strategies to achieve this goal.

\section{ACKNOWLEDGEMENT}

Authors take it as a privilege to acknowledge medical superintendent; NMCH and RC, HODs And Staffs of Department of Pharmacy Practice, Medicine Department, OBG, Orthopaedics, and Pediatrics for their support during the study.

\section{Conflict of Interest}

Authors declared that they have no conflict of interests.

\section{REFERENCES}

1. Lavakumar S, Jesurun JRS. A study on the level of drug compliance among the outpatients who are on a long-term drug therapy in a tertiary care teaching hospital at Kancheepuram district in Tamilnadu. Asian J Pharm Clin Res. 2017;10(3):174-76.

2. Shruthi R, Jyothi R, Pundarikaksha HP, Nagesh GN, Tushar TJ. A study of medication compliance in geriatric patients with chronic illnesses at a tertiary care hospital. J Clin Diagn Res. 2016;10(12):40-43.

3. Ahamad A, Mast RM, Nijpels G, Dekker MJ, Hugtenburg JG. Identification of drugrelated problems of elderly patients discharged from hospital. Arch Intern Med. 2014;8(3):155-65.

4. Srividya V, Balaje NRS. Adherence to medications among adults with diabetes mellitus in the urban field practice area of a private medical college in Bangalore, Karnataka, India. Int J Pub Health Res. 2019;6(5):200-206

5. Park HY, Seo SA, Yoo H, Lee K. Medication adherence and beliefs about medication in elderly patients living alone with chronic diseases. Arch Intern Med. 2018;12(3):175-181.

6. Nikolaus T, Kruse W, Bach M, Specht LN, Oster P, Schlierf G. Elderly patients' problems with medication. An in-hospital and follow-up study. Eur J Clin Pharmacol. 1996;49(7):255-59.

7. Sturgess IK, McElnay JC, Hughes CM, Crealey G. Community pharmacy based provision of pharmaceutical care to older patients. Pharm World Sci. 2003;25:218-26. 
8. Matche P, Manjunath D, Kotekar N, Pereira P. Assessment of prevalence and characteristics of pain and its association with quality of life in elderly. JORAPAIN. 2018;4(3):100-104

9. Yap AF, Thirumoorthy T, Kwan YH. Medication adherence in the elderly. J. Gerontol. Geriatr. 2016 Jun 1;7(2):64-7.

10. Gellad WF, Grenard JL, Marcum ZA. A systematic review of barriers to medication adherence in the elderly: looking beyond cost and regimen complexity. Am J Geriatr Pharmacother. 2011 Feb 1;9(1):11-23.

\section{AJPHR is}

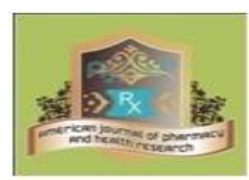

Peer-reviewed

monthly

Rapid publication

Submit your next manuscript at

editor@ajphr.com / editor.ajphr@gmail.com 